\title{
Flexible Patterning of Functional Microdot by Laser-Induced Dot Transfer
}

\author{
Aiko NARAZAKI ${ }^{* 1}$, Ryozo KUROSAKI ${ }^{* 1}$, Tadatake SATO ${ }^{* 1}$ and Hiroyuki NIINO*1 \\ ${ }^{* 1}$ Research Institute for Innovation in Sustainable Chemistry, National Institute of Advanced Indus- \\ trial Science and Technology (AIST), Central 5, 1-1-1 Higashi, Tsukuba, Ibaraki 305-8565, Japan \\ E-mail: Narazaki-aiko@aist.go.jp
}

\begin{abstract}
Laser-induced dot transfer (LIDT) is one of promising additive manufacturing techniques because it can realize flexible patterning of micron and submicron-sized dots even at atmospheric room-temperature conditions. In the LIDT process, a laser pulse is tightly focused onto a source film, leading to a transient melting of the film followed by sub-spot transfer using one-to-one microdot deposition with laser-illuminated area. Recently, we have developed a novel double-pulse LIDT process using the first pulse for preheating the source film and the second pulse for dot transfer. To investigate the double-pulse effect on microdot transfer of oxides, temporal high-temperature distributions of a thick indium tin oxide source film during double-pulse irradiation have been simulated using a Finite Element Method (FEM) approach. As a result, preheating by the first pulse decreased a difference in film temperature along both the film thickness and in-plane directions. This is effective to avoid film fracture, leading to high-quality patterning of a wide variety of microdots. DOI: $10.2961 /$ jlmn.2014.03.0015
\end{abstract}

Keywords: laser-induced dot transfer, flexible patterning, microdot, indium tin oxide, finite element method, laser-induced temperature distribution

\section{Introduction}

Laser-induced forward transfer (LIFT) is an attractive additive micro-patterning method because it can create micro-patterns by a simple atmospheric room-temperature process [1-3]. In general LIFT processes, the resolution of patterning is similar in size to the laser spot.

Over the last decade, laser-induced transfer of metal [47] and semiconducting [8-11] microdots has been reported using laser-induced transfer with one-to-one dot deposition and sub-spot resolution, which is referred to as laserinduced dot transfer (LIDT) [8-11] to distinguish it from conventional laser transfer techniques.

We have developed two types of LIDT processes with single-pulse and double-pulse laser irradiation. In the single-pulse LIDT, a single laser pulse is tightly focused onto a source film, which is on a supporting substrate, and sitecontrolled deposition of a single microdot on a receiver substrate placed behind the film occurs. The microdots have much smaller lateral dimensions than the laser focal area. The LIDT process is advantageous in that it has higher resolutions than commercially available inkjet techniques and there is an availability of solvent-free source materials.

Using our single-pulse LIDT, semiconducting $\beta-\mathrm{FeSi}_{2}$ microdot with a diameter of $2 \mu \mathrm{m}$ were successfully arrayed on a glass substrate as shown in Fig. 1(a-b) [8]. On the other hand, a possible mechanism of LIDT emerges from the discussion on morphology changes in source film, as follows. Figure 1(c) shows typical film morphology after the dot deposition; convex parts appeared corresponding to the laser-illuminated area. However, when there were small fluctuations in the fluence, unique microstructures with a flat disk-like top were sometimes observed.
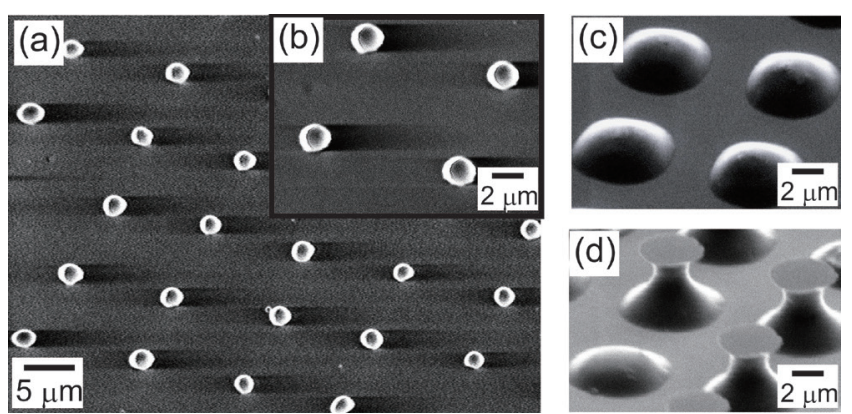

Fig. 1 (a-b) SEM photographs of $\mathrm{FeSi}_{2}$ microdots on a glass substrate deposited by a single-pulse LIDT. (c) A SEM image of $\mathrm{FeSi}_{2}$ source film after the successful transfer of dots. (d) A SEM image of the source film at an edge of the imaging area. Unique microstructures with a flat disk-like top appeared.

A possible mechanism for the unique microstructure in Fig. 1(d) is shown in Fig. 2. First, laser absorption at film causes transient heating and melting of the film. Then, the formation of a columnar-shaped jet with a diameter smaller than that of the laser spot occurs. When this jet touches the receiver substrate, the jet is cooled due to thermal conductance to the substrate. In the case of laser irradiation at an optimum fluence, the jet leaves a microdot on the substrate, as shown in Step 3A in Fig. 2. After the dot transfer, the remaining jet returns to the film surface, where solidification has already started, keeping the convex deformation. However, if laser fluence is lower than the optimum fluence for the microdot transfer, the laser-induced jet is solidified before returning to the film, resulting in the unique structure with a flat disk-like top in Step 3B in Fig. 2. 


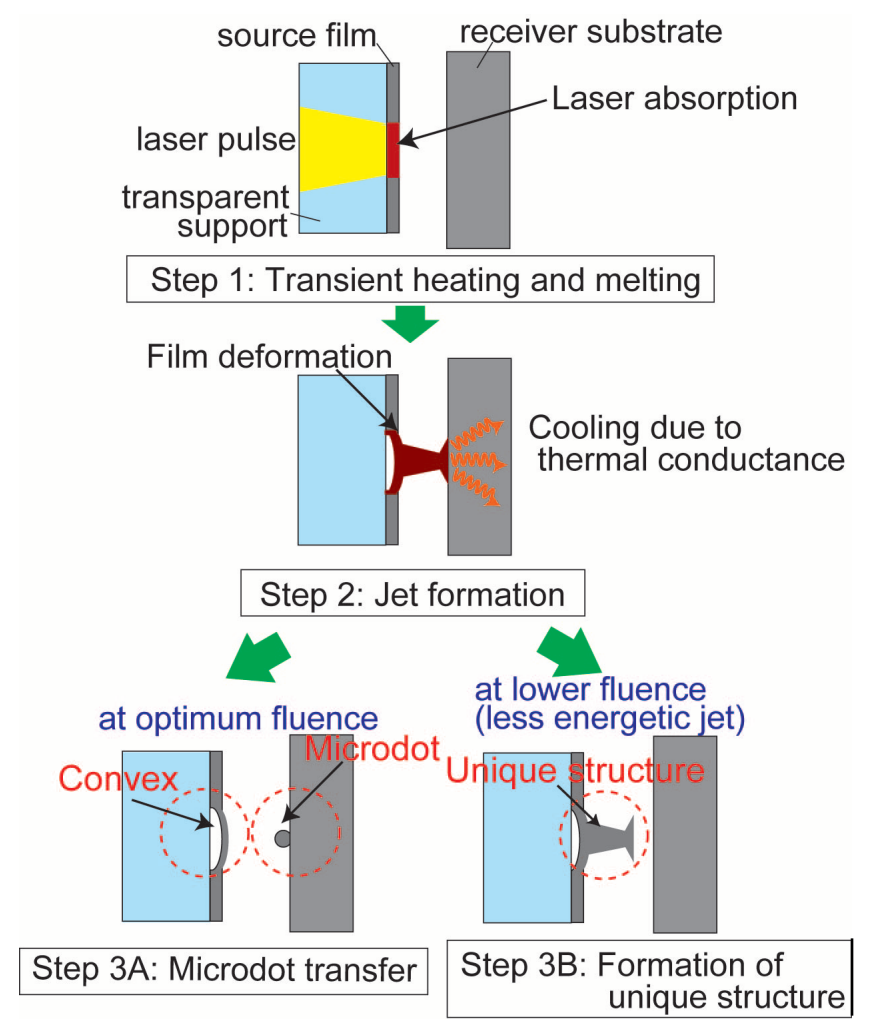

Fig. 2 A model for the microdot transfer by single-pulse LIDT. At an optimum fluence in Step 3A, a microdot is deposited onto a receiver substrate and a source film makes a convex. At lower fluence in Step 3B, there is no microdot transfer and the unique microstructure with a flat disk-like top appears on the source film.

However, in the case of oxide materials, it is somewhat difficult to deposit microdots by the single-pulse LIDT with high resolution and quality. In fact, in our previous attempt to apply LIDT to indium tin oxide (ITO), the lateral size of the transferred structure was never smaller than the laser spot size $[10,11]$. This problem of larger-sized film deposition like ITO microstructures in Fig. 3 (a) might
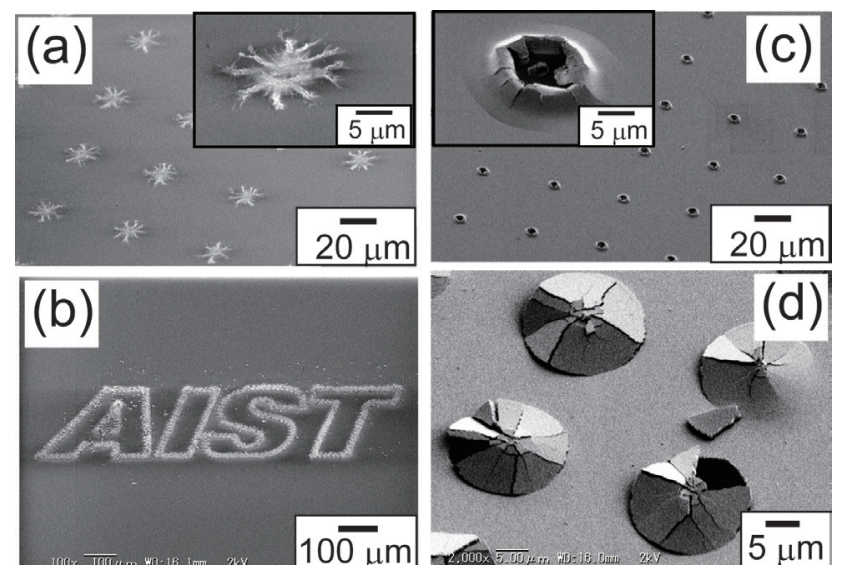

Fig. 3 (a-b) SEM photographs of ITO micro-patterns on a glass by a single-pulse laser transfer processes. (c) ITO source film after the transfer of (a). (d) $\mathrm{NiO}$ source film fractured after a single-pulse irradiation. (a)
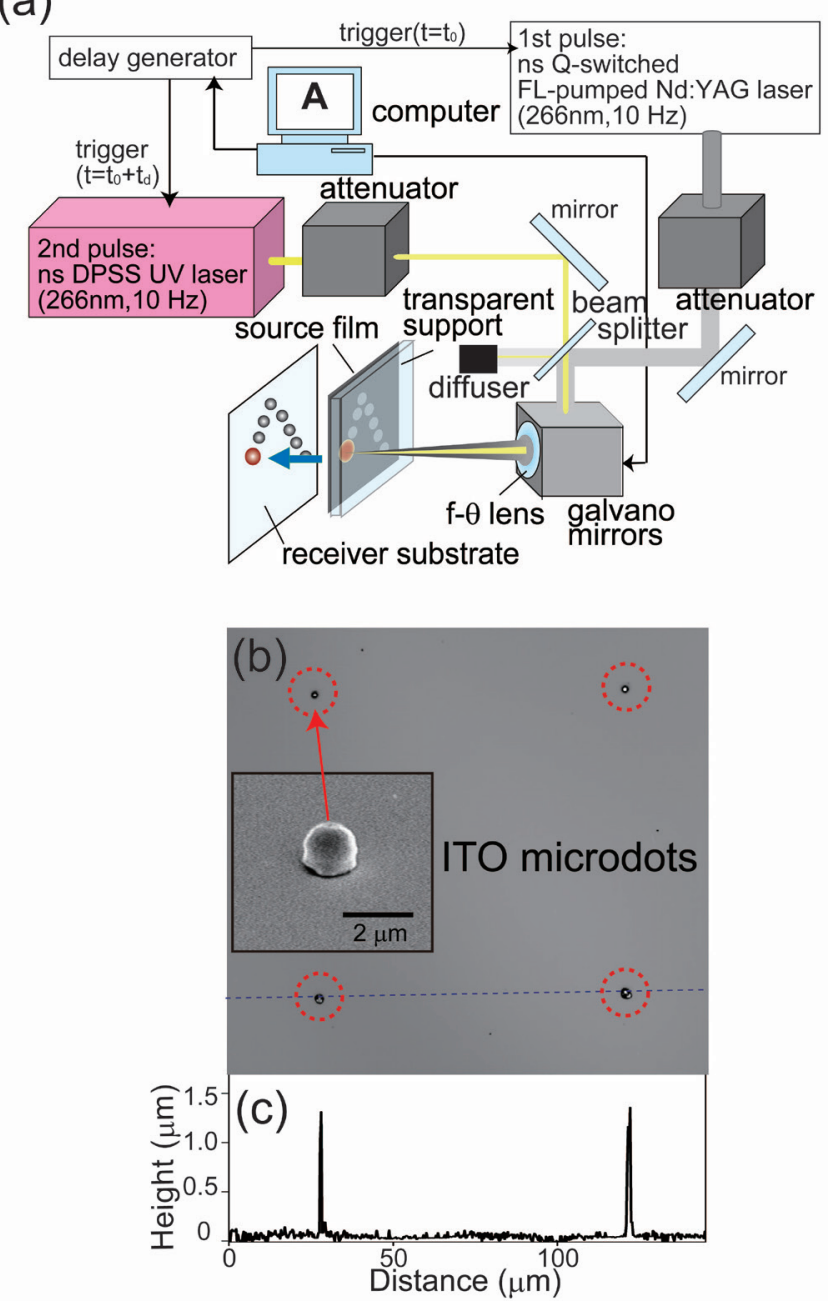

Fig. 4 (a) Typical setup for flexible patterning of functional microdots by double-pulse LIDT. (b-c) Confocal scanning laser microscopic image and profile of ITO microdots deposited by the double-pulse LIDT. The insert is a SEM image of the ITO dot.

stem from the difficulty in melting a source film entirely in the direction of a film thickness due to smaller thermal conductivity compared with metals. For the transfer of small spherical microdot by LIDT, it is important to melt a source film enough for the deposition of molten micronsized droplet onto a receiver. However, a partially-melted ITO film is peeled off by the receiving substrate in contact with the film in Fig. 3 (c). As a result, the transferred oxide structure had a lateral size similar with the laser spot and never became much smaller, different from LIDT of general metals. Furthermore, when an oxide film was irradiated by a laser pulse at higher energy to melt wholly in the film thickness direction, it often fractured after laser irradiation (Fig. 3(d)), emitting film flakes onto the receiver substrate and degrading the micro-patterns.

Recently, we have achieved the fabrication of a spherical ITO microdot by single-pulse LIDT with reducing thickness of source film [12]. However, the dot-vacancy still remained as a problem; single-pulse LIDT of oxides easily tends to make dot vacancies in micro-patterns. In order to reduce dot vacancies, we have developed a novel double-pulse LIDT process. So far, laser-induced forward 
transfer of film-like structures of some metals and metal oxides using femtosecond (fs) double pulses has been reported by Klini et al.[13] and Papadopoulou et al., respectively [14]. They irradiated the double pulses with equal intensity and diameter from the same fs laser light source. As a result, they found that the morphology and the size of the transferred spots were affected by the temporal delay of the double pulses separated by a picosecond timescale.

Different from the above works [13,14], our doublepulse LIDT system uses two types of ns laser pulses with different diameters from separate light source. Figure 4 (a) shows a typical setup of our double-pulse LIDT system. Two types of light sources were used. The beam splitter with transmission and reflection light branch ratio of 50:50 was used as a beam mixer. The first ns pulse was irradiated onto a transparent support / source film interface through
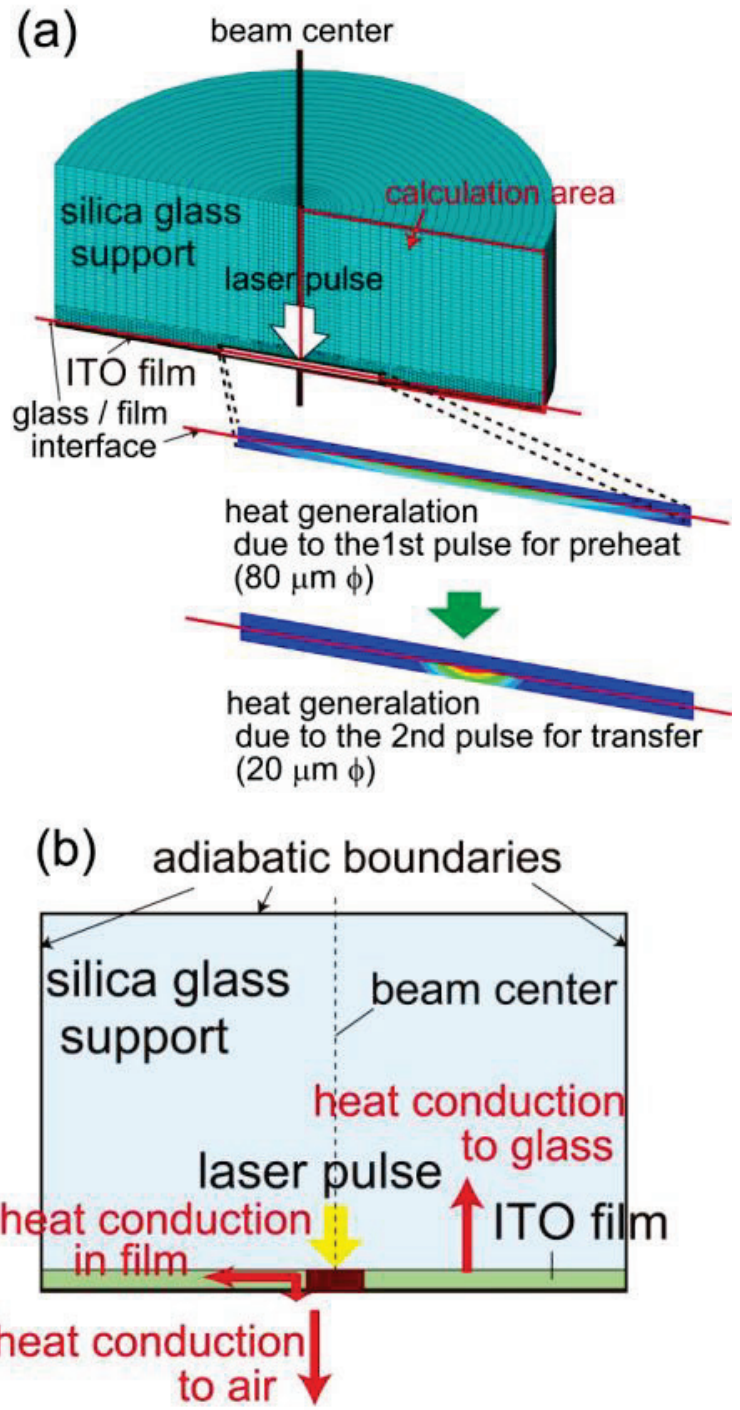

Fig. 5 (a) FEM mesh for ITO source film on silica glass support irradiated by double laser pulses during the LIDT process. The first and second pulses have a diameter of 80 and $20 \mu \mathrm{m}$, respectively. The time delay between them was $500 \mathrm{~ns}$. The laser pulses are absorbed only in the ITO films and become a heating source. (b)Schematic diagram of dissipation of generated heat and boundary conditions. the support to preheat the film. After adequate time delay, the second ns pulse was more tightly focused on the same position for microdot transfer [15]. As a result, an array with spherical ITO microdots was patterned with reducing dot vacancies, as shown in Fig. 4 (b).

In this work, to make clear the effect of our doublepulse LIDT, laser-induced temperature distributions during the double-pulse laser irradiation were simulated using finite element method (FEM) techniques. Especially, we focused on a thick ITO film because the LIDT of thick oxide films is still more difficult than thinner ones and is crucial for the flexible patterning over a wide dot size range.

\section{Experimental}

FEM simulations were employed to investigate temporal change of laser-induced temperature distribution in an oxide source film during the double-pulse LIDT. As a model case, ITO films with a thickness of $1200 \mathrm{~nm}$ were used as a source film.

When a nanosecond laser pulse is absorbed in a material, the energy transfer is governed by the heat diffusion equation, which is based on Fourier's law of conduction:

$$
\rho c_{p} \frac{\partial T}{\partial t}=k \nabla^{2} T+\dot{q}
$$

where $\rho, c_{p}, k$ and $\dot{q}$ are density, specific heat, thermal conductivity and a rate of heat generation due to the laser light absorption, respectively.

In UV region above $266 \mathrm{~nm}$, since laser light absorption in a silica glass support is negligible, the laser pulse is absorbed in the ITO film. As a result, the heat diffusion equation can be separated into two equations for the absorbing ITO film and the transparent silica glass support:

$$
\begin{aligned}
& \rho_{f} c_{p f} \frac{\partial T_{f}}{\partial t}=k_{f} \nabla^{2} T_{f}+\dot{q} \\
& \dot{q}=-\left(1-R_{1}\right)\left(1-R_{2}\right) \frac{d I(z, t)}{d z} \\
& \quad=\left(1-R_{1}\right)\left(1-R_{2}\right) \alpha I_{0}(t) e^{-\alpha z} \quad 0 \leq t \leq t_{p} \\
& \rho_{s} c_{p s} \frac{\partial T_{s}}{\partial t}=k_{s} \nabla^{2} T_{s}
\end{aligned}
$$

where the subscripts of $\mathrm{f}$ and $\mathrm{s}$ indicate the regions in the film and support, respectively. $R_{1}$ and $R_{2}$ are the reflectivity at air/glass and glass/film interfaces, respectively. $I_{0}(\mathrm{t})$ is the local laser power density at the glass surface and $t_{\mathrm{p}}$ is the irradiation time, namely the length of the laser pulse. $\alpha$ is the absorption coefficient of the ITO film.

FEM simulations of the temporal high-temperature distributions in the ITO source films were performed using Ansys (version 12.0). A three-dimensional finite element model with a diameter of $240 \mu \mathrm{m}$ and a height of $90 \mu \mathrm{m}$ was developed to simulate the silica glass/ITO film interface as shown in Fig. 5 (a). Since the model was symmetric and subjected to a symmetric laser pulse that is both spatially and temporally Gaussian in shape, only one half of the cross-sectional plane along the incident laser direction was considered for computational efficiency. 
Different from our previous simulation for a thinner ITO film (with a thickness of $250 \mathrm{~nm}$ ) where both the first and second pulses assumed to be $266 \mathrm{~nm}$, the first and second ones were 355 and $266 \mathrm{~nm}$, respectively, in this work. The ITO film has light absorption coefficients of $7.2 \times 10^{6}$ and $2.1 \times 10^{6} \mathrm{~m}^{-1}$ at 266 and $355 \mathrm{~nm}$, respectively. Thus, the first laser pulse at $355 \mathrm{~nm}$ is effective to preheat the ITO film deeper from the support/film interface and more uniformly along the laser incident axis. Here, it should be mentioned that the above light absorption coefficients are values at room temperature. The temperature dependence of the light absorption coefficient of ITO is unknown to the authors, and is neglected in the calculation in this work. The absorption coefficient of solid ITO is used for liquid. The diameters of first and second laser pulses and the time delay between them used for FEM simulations are set to be similar with our previous experimental conditions for the double-pulse LIDT [13]. The diameters of the first and second laser pulses were 80 and $20 \mu \mathrm{m}$, respectively. The time delay between them was $500 \mathrm{~ns}$.

Figure 5 (b) shows a schematic diagram of the dissipation processes of the generated heat and the boundary conditions for the simulation. In addition to Eq. (2), the heat radiation from the surface was taken into account for the ITO film. The emissivity was assumed to be an average value of 0.5 . To simplify the simulation, the silica glass support and ITO source film were assumed to be isotropic, and only the ITO phase change from solid to melt is taken into account as an enthalpy change of $83.74 \mathrm{~kJ} \cdot \mathrm{mol}^{-1}$ around the melting temperature of $2186 \mathrm{~K}$ [16]. The density, temperature, specific heat, and thermal conductivity values of ITO and silica glass used in our simulation are listed in Table 1 [16-18].

Table 1 The physical properties of ITO and silica glass.

\begin{tabular}{lcrrr}
\hline & $\begin{array}{c}\rho \\
\left(\mathrm{kg} \cdot \mathrm{m}^{-3}\right)\end{array}$ & \multicolumn{1}{c}{$\begin{array}{c}T \\
(\mathrm{~K})\end{array}$} & $\begin{array}{c}c_{p} \\
\left(\mathrm{~J} \cdot \mathrm{kg}^{-1} \cdot \mathrm{K}^{-1}\right)\end{array}$ & $\begin{array}{c}k \\
\left(\mathrm{~W} \cdot \mathrm{m}^{-1} \cdot \mathrm{K}^{-1}\right)\end{array}$ \\
\hline ITO $^{\text {a) }}$ & & 300 & 362 & \\
& \multirow{2}{*}{7180} & 1000 & 463 & 5.95 \\
& & 2000 & 493 & \\
\hline Silica glass & & 300 & 692 & 1.38 \\
& 2200 & 500 & 1049 & 1.62 \\
& & 1200 & 1130 & 2.17 \\
& & 1600 & 1249 & 4.00 \\
\hline
\end{tabular}

a) The density, $\rho$, and specific heat, $c_{p}$, substitute available values of $\mathrm{In}_{2} \mathrm{O}_{3}$ for those of ITO.

\section{Results and discussion}

\subsection{FEM simulation for single-pulse LIDT}

Figure 6 (a) shows temporal change of the ITO film temperature at the center of laser beam after single-pulse incidence simulated by FEM. The laser pulse energy was 2 $\mu \mathrm{J} \cdot$ pulse $^{-1}$. The ITO film temperatures at glass/film interface and film surface are denoted by the red and blue curves, respectively. The green curve stands for the timing of laser pulse irradiation (fwhm $30 \mathrm{~ns}$, beam diameter of 20 $\mu \mathrm{m})$. The laser pulse rapidly increased ITO film tempera-
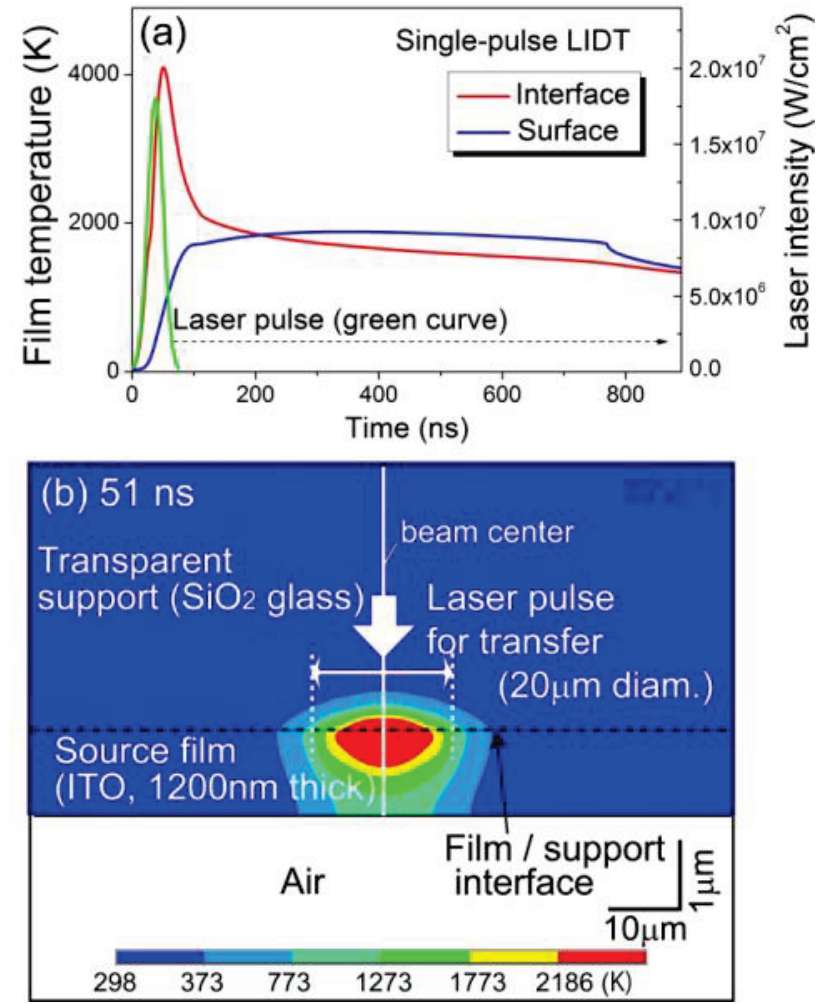

Fig. 6 Temporal change and distribution of the ITO film temperature at single-pulse irradiation simulated by FEM. (a) Temporal change of the film temperature at the film/glass interface (red curve) and film surface (blue curve). The green curve corresponds to a timing of laser pulse. (b) Temperature distribution at $51 \mathrm{~ns}$ after laser pulse when the temperature at the film/support interface reached a maximum.

ture at the interface where the laser pulse was incident. The film temperature at the interface reached a maximum over the ITO melting temperature of $2186 \mathrm{~K}$ at $51 \mathrm{~ns}$ after the pulse. At the same time, the film surface temperature was around $800 \mathrm{~K}$. Here, it is noteworthy that the film temperature at the interface became lower than that at the surface due to heat conduction to the adjacent glass support after 200 ns.

Figure 6 (b) shows the FEM simulated temperature distribution in the ITO film at $51 \mathrm{~ns}$ when the film temperature at the interface reached a maximum. The temperature in the red-colored part exceeds the ITO melting temperature. Thus, only the film near the film/glass interface had a temperature above the ITO melting temperature, resulting in a partial melting along the film thickness direction.

\subsection{FEM simulation for double-pulse LIDT}

Figure 7 (a) shows a temporal change in ITO film temperature at the beam center during double-pulse irradiation. The time delay between the double pulses (green curves) was set at $500 \mathrm{~ns}$. The laser pulse energy of the first pulse and second pulse was 5 and $1.75 \mu \mathrm{J} \cdot$ pulse $^{-1}$, respectively, which was determined by reference to our previous doublepulse LIDT experiments [15]. The first pulse for preheat 

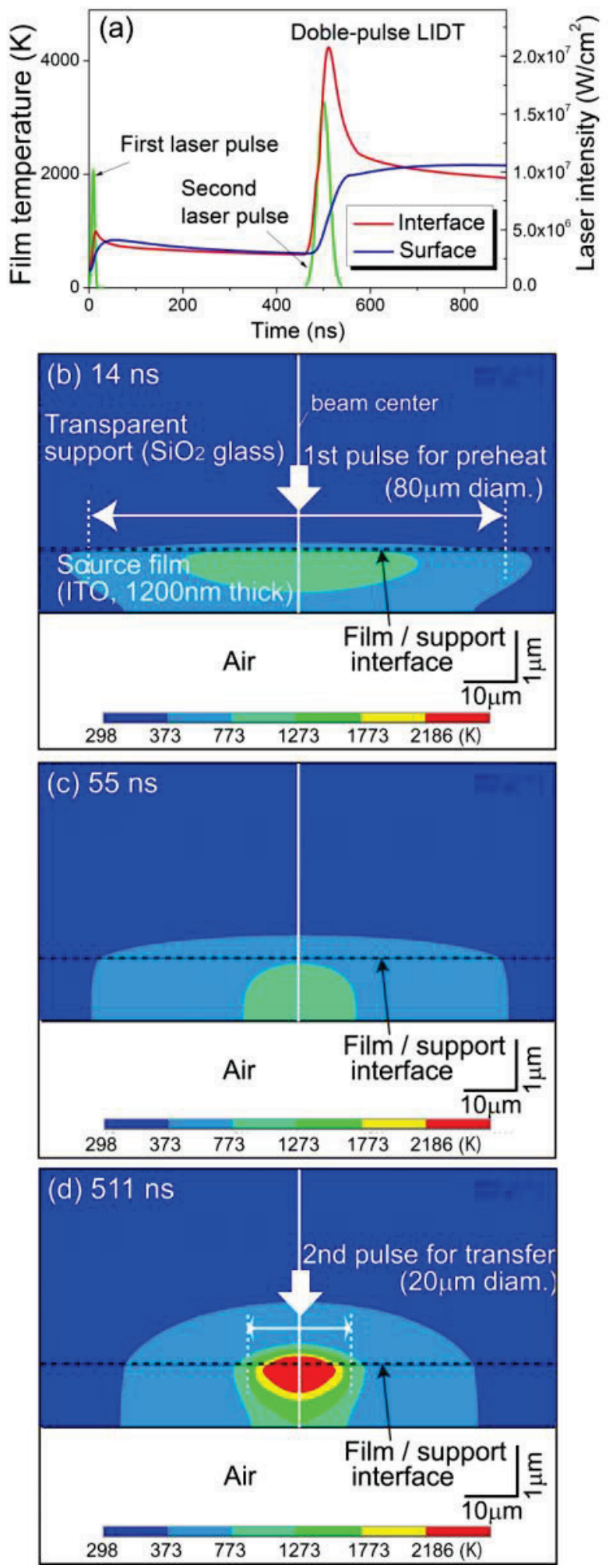

Fig. 7 Temporal change and distributions of the ITO film temperature at double-pulse LIDT. (a) Temporal change at the interface (red curve) and film surface (blue curve). The timing of the first and second pulses (green curves) is shown. (b-d) Temperature distributions at (b) $14 \mathrm{ns,} \mathrm{(c)} 55 \mathrm{~ns}$, and (d) $511 \mathrm{~ns}$.

(fwhm $7 \mathrm{~ns}$, beam diameter of $80 \mu \mathrm{m}$ ) increased the film temperature up to a maximum of about $1000 \mathrm{~K}$ at the glass/film interface. At $55 \mathrm{~ns}$ the film surface temperature reached a high-temperature peak. Then, such hightemperature distribution was kept until incidence of the second pulse for transfer (fwhm $30 \mathrm{~ns}, 20 \mu \mathrm{m}$ ). At $511 \mathrm{~ns}$, the film temperature at the interface reached a maximum and over the ITO melting temperature. At the same time, the film surface temperature was about $1000 \mathrm{~K}$, which is higher than $800 \mathrm{~K}$ during single-pulse LIDT in Fig. 6 (a). Therefore, preheating by the first pulse is effective to diminish a difference in film temperature along the film thickness direction.

Laser-induced temperature distributions during doublepulse irradiation are demonstrated at (b) $14 \mathrm{ns,} \mathrm{(c)} 55 \mathrm{~ns}$ and (d) $511 \mathrm{~ns}$ in Fig. 7. In comparison with the singlepulse irradiation in Fig. 6 (b), the first pulse with large beam diameter has a striking effect on preheating a large area along film in-plane direction. Thus, preheating by the first pulse is effective to diminish a difference in film temperature along both the film thickness and in-plane directions.

As addressed in the Introduction section, it is important to melt the whole part along the film thickness direction and reduce the difference in film temperature and resultant thermal stress for the LIDT of oxide materials. To realize melting in the entire part along the film thickness direction, further optimization such as pulse energy is under progress. Anyway, the double-pulse LIDT is a promising technique for flexible micro-patterning of a variety of materials including even oxides, because it can control laser-induced high-temperature and thermal-stress in a source film during the LIDT process more precisely

\section{Conclusion}

In order to clarify the double-pulse effect during LIDT, high-temperature distributions in ITO source films caused by the double-pulse laser irradiation were simulated using FEM. As a result, preheating by the first pulse diminished a difference in film temperature along both the film thickness and in-plane directions, compared with only a single-pulse irradiation. The reduction in the temperature difference and resultant thermal stress is useful to avoid the fracture of source films, resulting in high-quality patterning of microdots. Therefore, flexible patterning of a variety of functional microdots can be realized with double-pulse LIDT, and this is promising for future optoelectronic integrations.

\section{References}

[1] J. Bohandy, B. F. Kim and F. J. Adrian: J. Appl. Phys., 60, (1986) 1538.

[2] A. Piqué, D. B. Chrisey, R. C. Y. Auyeung, J. FitzGerald, H. D. Wu, R. A. McGill, S. Lakeou, P. K. Wu, V. Nguyen and M. Duignan: Appl. Phys. A, 69, (1999) S279.

[3] Y. Nakata and T. Okada: Appl. Phys. A, 69, (1999) S275.

[4] D. A. Willis and V. Grosu: Appl. Phys. Lett., 86, (2005) 244103.

[5] D. P. Banks, C. Grivas, J. D. Mills, R. W. Eason and I. Zergioti: Appl. Phys. Lett., 89, (2006) 193107.

[6] A. I. Kuznetsov, R. Kiyan and B. N. Chichkov: Opt. Express, 18, (2010) 21198.

[7] A. I. Kuznetsov, C. Unger, J. Koch and B. N. Chichkov: Appl. Phys. A, 106, (2012) 479. 
[8] A. Narazaki, T. Sato, R. Kurosaki, Y. Kawaguchi and H. Niino: Proc. of SPIE, 6458, (2007) 645814.

[9] A. Narazaki, T. Sato, R. Kurosaki, Y. Kawaguchi and H. Niino: Appl. Phys. Express, 1, (2008) 57001.

[10]A. Narazaki, T. Sato, R. Kurosaki, Y. Kawaguchi and H. Niino: Appl. Surf. Sci., 255, (2009) 9703.

[11] A. Narazaki, R. Kurosaki, T. Sato, Y. Kawaguchi, W. Watanabe and H. Niino: J. Laser Micro/Nanoengineering. 7, (2012) 77.

[12]A. Narazaki, R. Kurosaki, T. Sato and H. Niino: Appl. Phys. Express, 6, (2013) 92601.

[13]A. Klini, P. A. Loukakos, D. Gray, A. Manousaki and F. Fotakis: Opt. Exp., 16, (2008) 11300.
[14]E. L. Papadopoulou, E. Axente, E. Magoulakis, C. Fotakis and P. A. Loukakos: Appl. Surf. Sci., 257, (2010) 508.

[15]A. Narazaki, R. Kurosaki, T. Sato, Y. Kawaguchi and H. Niino: J. Laser Micro/Nanoengineering. 9, (2014) 10.

[16]G. V. Samsonov: "The Oxide Handbook" (Plenum, New York, 1982) pp. 54, 61, 101.

[17] "Thermophysical Properties Handbook" ed. by Japan Society of Thermophysical Properties (Yokendo, Japan, 2008) pp. 272, 300 (in Japanese).

[18] T. Ashida, A. Miyamura, N. Oka, Y. Sato, T. Yagi, N. Taketoshi, T. Baba and Y. Shigesato: J. Appl. Phys., 105, (2009) 073709.

(Received: June 12, 2014, Accepted: November 04, 2014) 